\title{
Teaching Hints
}

The present textbook addresses students of Mathematics, Computer Science and Science covering typical material for introductory courses in Numerical Analysis with clear emphasis towards Scientific Computing.

We start with Gaussian elimination for linear equations as a classical algorithm and discuss additional devices such as pivoting strategies and iterative refinement. Chapter 2 contains the indispensable error analysis based on the fundamental ideas of Wilkinson. The condition of a problem and the stability of algorithms are presented in a unified framework and exemplified by illustrative cases. Only the linearized theory of error analysis is presented - avoiding, however, the typical " $\varepsilon$-battle". Rather, only differentiation is needed as an analytical tool. As a specialty we derive a stability indicator which allows for a rather simple classification of numerical stability. The theory is then worked out for the case of linear equations, thus supplying a posteriori a deeper understanding of Chapter 1 . In Chapter 3 we deal with methods of orthogonalization in connection with linear least squares problems. We introduce the extremely useful calculus of pseudoinverses, which is then immediately applied in Chapter 4 . There, we consider iterative methods for systems of nonlinear equations (Newton's method), nonlinear least squares problems (Gauss-Newton method) and parameter-dependent problems (continuation methods) in close mutual connection. Special attention is given to the affine invariant form of the convergence theory and the iterative algorithms. A presentation of the power method (direct and inverse) and the QR-algorithm for symmetric eigenvalue problems follow in Chapter 5. The restriction to the real symmetric case is motivated from the beginning by a condition analysis of the general eigenvalue problem. In this context the singular value decomposition fits perfectly, which is so important in applications.

After the first five rather closely connected chapters the remaining four chapters also comprise a closely connected sequence. The sequence begins in Chapter 6 with an extensive treatment of the theory of three-term recurrence relations, which play a key role in the realization of orthogonal projections in function spaces. Moreover, the significant recent spread of symbolic computing has renewed interest in special functions also within Numerical Analysis. 
The condition of three-term recurrences is presented via the discrete Green's function. Numerical algorithms for the computation of special functions are exemplified for spherical harmonics and Bessel functions. In Chapter 7 classical interpolation and approximation in the one-dimensional special case are presented first, followed by non-classical methods like Bézier techniques and splines, which nowadays play a central role in CAD (Computer Aided Design) or CAGD (Computer Aided Geometric Design), i.e. special disciplines of computer graphics. Our presentation in Chapter 8, which treats iterative methods for the solution of large symmetric linear equations, is conveniently based on Chapter 6 (three-term recurrences) and Chapter 7 (min-max property of Chebyshev polynomials). The same is true for the Lanczos algorithm for large symmetric eigenvalue problems. The final Chapter 9 turns out to be a bit longer: it carries the bulk of the task to explain principles of the numerical solution of differential equations by means of the simplest problem type, which here is numerical quadrature. After the historical Newton-Cotes formulas and the Gauss quadrature, we progress towards the classical Romberg quadrature as a first example of an adaptive algorithm, which, however, only adapts the approximation order. The formulation of the quadrature problem as an initial value problem opens the possibility of working out a fully adaptive Romberg quadrature (with order and stepsize control) and at the same time a didactic first step into extrapolation methods, which play a prominent role in the solution of ordinary differential equations. The alternative formulation of the quadrature problem as a boundary value problem is exploited for the derivation of an adaptive multigrid algorithm: in this way we once more present an important class of methods for ordinary and partial differential equation in the simplest possible case.

For a typical university term the contents of the book might be too rich. For a possible partitioning of the presented material into two parts we recommend the closely connected sequences Chapter $1-5$ and Chapter $6-9$. Of course, different "teaching paths" can be chosen. For this purpose, we give the following connection diagram: 


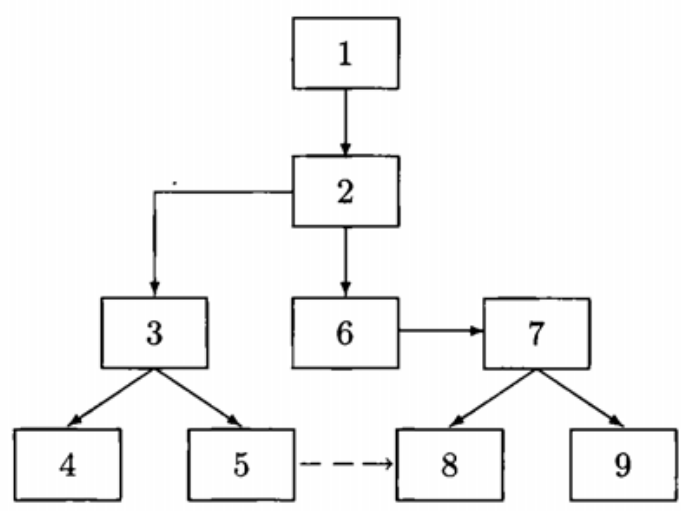

As can be seen from this diagram, the chapters of the last row (Chapters $4,5,8$, and 9) can be skipped without spoiling the flow of teaching according to the personal scientific taste. Chapter 4 could be integrated into a course on "Nonlinear optimization", Chapters 5 and 8 into a course on "Numerical linear algebra" or Chapter 9 into "Numerical solution of differential equations".

At the end of each chapter we added exercises. Beyond these explicit exercises further programming exercises may be selected from the numerous algorithms, which are given informally (usually as pseudocodes) throughout the textbook. All algorithms mentioned in the text are internationally accessible via the electronic library $e$ Lib of the Konrad Zuse Center. In the interactive mode $e L i b$ can be reached via:

Datex-P: $\quad+45050331033($ WIN) +2043623331033 (IXI)

INTERNET: elib.ZIB-berlin.de (130.73.108.11)

login: $\quad$ elib (no password necessary)

In addition, there is the following e-mail access:

X.400: $\quad \mathrm{S}=\mathrm{eLib} ; \mathrm{OU}=\mathrm{sc} ; \mathrm{P}=\mathrm{ZIB}-\mathrm{Berlin} ; \mathrm{A}=\mathrm{dbp} ; \mathrm{C}=\mathrm{de}$

INTERNET: elib@elib.ZIB-Berlin.de

BITNET: $\quad$ eLib@sc.ZIB-Berlin.dbp.de

UUCP: $\quad$ unido!sc.ZIB-Berlin.dbp.de!eLib

Especially for users of Internet there is an "anonymous ftp" access (elib.ZIBBerlin.de - 130.73.108.11) . 